\title{
Vendor Name
}

National Cancer Institute

\section{Source}

National Cancer Institute. Vendor Name. NCI Thesaurus. Code C117200.

The literal identifier of the vendor that performs an assessment or examination. 